\title{
Judgments of shape orientation: A matter of contrasts
}

\author{
LILA G. BRAINE, EILEEN PLASTOW, and SHARON L. GREENE \\ Barnard College, Columbia University, New York, New York
}

\begin{abstract}
A model is presented of the way in which judgments of the orientation of shapes may be made. It is proposed that orientation judgments can be made on different bases and at varying degrees of precision; three levels of precision are defined. A series of four experiments using a reactiontime paradigm explored the predictions of the model, and support for the three kinds of judgments was obtained. In addition, symmetry played a significant role only for those judgments in which the orientation of the top-bottom axis of the shape served to differentiate among orientations. The results are discussed in terms of their implications for judgments of shape orientation in general and left-right judgments in particular.
\end{abstract}

Work on the relationship between orientation and shape has been concerned with two broad problems. One is the question of how the recognition of a shape is affected by rotation of the shape, and Rock (1973) has reported a definitive set of studies on this issue. The other area, into which the present work falls, is concerned with how the orientation of shape itself is identified. Work on this problem has tended to ask whether orientation is judged with respect to the retinal or the environmental framework (e.g., Attneave \& Olson, 1967; Braine \& Knox, 1975), and to examine the basis for the greater difficulty in making left-right judgments of orientation than up-down ones (e.g., Corballis \& Beale, 1976). The present work, on the other hand, asks directly about the basis, or bases, for making judgments of the orientation of shapes, regardless of the framework used.

It is not clear whether an explicit theory exists of how judgments of shape orientation are made. One view has been offered by Howard (1982): "When a person judges the orientation of an object, he relates the direction of some recognizable axis in the object, an intrinsic axis, to some axis of reference outside the object, an extrinsic axis" (p. 521, author's italics). For objects with a defined top and bottom, such as natural objects with a customary upright, the "direction" of the axis would presumably include information on the location of the top feature. Other views (e.g., Farrell, 1979, p. 10; Maki, Grandy, $\&$ Hauge, 1979 , p. 53) have focused more on the judgment of relative position or location of particular features.

This work was supported by NICHD Grant HD-09513. The manuscript was prepared while the senior author was a Fellow at the Center for Advanced Study in the Behavioral Sciences; financial support there from the Alfred P. Sloan Foundation is gratefully acknowledged. We would like to thank Martin D. S. Braine, Celia Fisher, and Robert Remez for their helpful comments on the manuscript. The second author is currently at the University of Surrey, England, and the third author is at Bell Communications Research, Morristown, New Jersey. Reprints may be obtained from Lila G. Braine, Department of Psychology, Barnard College, 3009 Broadway, New York, NY 10027-6598.
In this context, the greater difficulty in making left-right than up-down judgments has been attributed to a greater difficulty in encoding direction or position of features on the horizontal than on the vertical dimension (e.g., Farrell, 1979). In general, these views imply that all judgments of shape orientation are made in the same way, essentially by an implicit description of the relationship between some aspect of the shape and the vertical and horizontal axes of environmental space.

The present work offers an alternative view, and starts with some observations that have not been discussed in much of the work on orientation perception. First, some orientation judgments have a normative character. Everyday objects with a customary position in space and alphanumeric shapes are seen as upright or nonupright. Judgments of uprightness and nonuprightness are made for works of nonrepresentational art (Arnheim, 1954) and for geometric shapes (e.g., Ghent, 1961). Furthermore, a normative judgment is made with respect to left and right when letters are described as "normal" or "reversed" in mental rotation tasks. The normative aspect of judgments has not been included in the ways (referred to above) in which orientation judgments have been conceptualized. Second, it is a common judgment to describe a shape as turned to the side. If the perception of sideways is an abstraction derived from prior experience of identifying the shape as turned to one or the other side, then identifying a shape as turned to a particular side should be easier than the more abstract identification that a figure is turned sideways. However, the errors made by children indicate that identifying sideways is easier than identifying left and right (e.g., Corballis \& Beale, 1976, chap. 10).

The present work assumes that people judge the orientation of a shape in different ways, that these ways vary in difficulty and complexity, and that the ensuing judgments provide information that varies in precision (Braine, 1978). For the simplest judgment, a shape is judged merely as upright or nonupright. That is, in identifying 
the orientation of figures that are upright and upside down, or upright and sideways, the upside-down or sideways figures need only be identified as nonupright, and not specifically as upside down or sideways. For natural shapes, uprightness could be based on a match between features of the input and a representation of the item in long-term memory. Nonuprightness would be indicated by a mismatch, and virtually any feature could signal the mismatch. Cooper and Shepard (1973, p. 90) have made a comparable assumption in their work with alphanumeric material. Uprightness and nonuprightness could thus be judged without necessarily identifying the position of the top-bottom axis or of the top feature.

At the next degree of difficulty, nonupright figures are identified as being specifically upside down or sideways by identifying the top-bottom axis as horizontal or vertical. In this view, a shape can be identified as specifically upside down, or as specifically sideways, without reference to the position of the top feature of the figure. The orientation of the top-bottom axis of a phenomenally nonupright shape does, in itself, provide enough information to identify the shape as upside down or as sideways, and presumably as diagonal as well (see Braine, 1978).

The third level of complexity and specificity for orientation judgments is exemplified by the identification of a sideways shape as turned to a particular side (in the context of stimuli turned to both the left and right). This judgment can be made only by encoding the relationship between the top feature of a phenomenally sideways shape and a side of the framework. That is, the judgment requires the selection of a specific feature and an implicit description of its relationship to a spatial referent. This relational judgment seems to be the same as the judgment that traditional thinking assumes to be the basis for all judgments of shape orientation. Judgments that an upright shape is facing to the left or the right would also be made at the third level of difficulty, because the judgments require encoding of the relationship between the front feature and a side of the framework. Furthermore, any noncategorical judgment of tilt, for example, judging that a shape is rotated $35^{\circ}$, would involve this kind of relational judgment.

It is easy to see how the three kinds of judgment apply to natural objects and alphanumeric shapes; however, there are reasons for believing that this approach to orientation judgments applies to geometric shapes as well. First, there is a moderate body of data showing that many geometric shapes elicit consistent judgments of uprightness and nonuprightness in children and adults in the United States (e.g., Ghent, 1961; Pick, Klein, \& Pick, 1966; Schaller \& Harris, 1975) and in children in Iran (Antonovsky \& Ghent, 1964). Second, Rock (1973) has made the general claim that all shapes are assigned a top, bottom, and sides in coding the description of a shape. In this view, even geometric shapes without an intrinsic top may be said to have an apparent upright because of an assigned top and the consequent specification of a topbottom axis.

The proposal that orientation judgments can be made on three different bases (resulting in three different kinds of judgments) has implications for understanding the nature of the greater difficulty of making left-right than updown judgments of shape orientation. The usual approaches to this problem have assumed, in one form or another, that the issue is to explain why judgments of position or direction are more difficult to make for the horizontal than for the vertical axis; that is, why features at the left and right of a shape are more confusable than features at the top and bottom (e.g., Farrell, 1979; Maki et al., 1979). However, the present proposal implies that left-right judgments are more difficult because they require a relational judgment to be made, which is assumed to be more complex cognitively than the match-mismatch process underlying the upright-nonupright judgment made to upright and upside-down stimuli. Recent work is compatible with this focus on the cognitive processes underlying tasks as a way of understanding the differences between left-right and upright-nonupright judgments.

First, there is a considerable literature that shows that the relative difficulty of left-right and upright-nonupright tasks is very sensitive to procedural factors. Presenting stimuli one at a time decreases dramatically the difficulty of left-right discrimination tasks for preschool children (Fisher, 1979); aligning two stimuli one above the other, in contrast to side by side, decreases the left-right difficulty for both kindergarten children and adults (e.g., Huttenlocher, 1967; Staller \& Sekuler, 1976); other procedural factors have also been found to influence relative task difficulty (e.g., Corballis \& Beale, 1976; Fisher, 1982; Howard, 1982). Second, a number of studies have shown that it is not necessarily more difficult to encode the location of features along the horizontal axis than to encode such information along other axes (Maki et al., 1979; Rock, Di Vita, \& Barbeito, 1981; Sholl \& Egeth, 1981; Vogel \& Behrend, 1982). The marked effect of procedural variables underlines the importance of analyzing the cognitive strategies underlying the responses made in different tasks (see also Greene, Plastow, \& Braine, 1985). This conclusion contrasts with the more usual assumption, in theoretical discussions of the difficulty of left-right tasks, that analysis should focus on the characteristics of the horizontal axis of space (e.g., Farrell, 1979) or the symmetry of the body (e.g., Corballis \& Beale, 1976).

To recapitulate, identifying the orientations of upright and upside-down shapes presented in the same context can be reconceptualized as identifications of uprightness and nonuprightness, as can judgments of upright and sideways orientations (Level 1). The identification of a shape as being specifically upside down or sideways can be based on identification of the top-bottom axis of a nonupright shape as being vertical or horizontal (Level 2). A left- 
right judgment requires encoding the relationship between a particular feature of a sideways shape and a side of a spatial framework (Level 3). ${ }^{1}$ In this model, it is not position on the vertical or horizontal axis that determines the difficulty of an orientation judgment; rather, difficulty is related to the cognitive complexity of the judgment, which is assumed to increase from Level 1 to Level 3. The basis for making a particular judgment (i.e., the kind of judgment made) will depend on the orientation contrasts available in a task, since the contrasts determine the strategy (or level) used for a given set of judgments. For all levels, the judgments are seen as being based on an abstract property of the shape, that is, involving the identification of nonuprightness, leftness, and so on, in a wide range of shapes.

There is empirical support for the first two degrees of difficulty of the model from work with children (Braine, 1978; Braine, Lerner, \& Relyea, 1980) and infants (Fagan \& Shepherd, 1979). The present work explores systematically the model's predictions for adult judgments of orientation.

\section{EXPERIMENT 1}

The first two levels of difficulty were investigated by asking whether the time to identify a shape as being upside down was faster in the context of upright shapes (Level 1) than in the context of sideways shapes (Level 2), and, analogously, by asking whether the time to identify sideways was faster in the context of upright than of upside-down shapes. If judgments of the orientation of single shapes were made by relating an intrinsic aspect of the shape to an extrinsic framework, then the orientation of the other stimuli in the block of trials should have no effect on judgment time. On the other hand, if judgment time depended on the orientation contrasts offered, then upside down (and sideways) should be judged on different bases in the two conditions. An earlier study had found that the upright-nonupright conditions were associated with faster RTs than were the upside-downsideways conditions, but judgment times had interacted with the hand used to signal the responses (Braine, Relyea, \& Davidman, 1981). To eliminate these interactions, the present study used verbal, rather than manual, responses.

Also, some data in the prior experiment had suggested that symmetry about the top-bottom axis might influence judgment time. According to the proposed model, any factor contributing to identification of the top-bottom axis should facilitate judgments based on perception of that axis (Level 2), and there are two reasons for expecting symmetry to facilitate such perception. First, symmetry is processed early, and the axis of symmetry is perceived before other features of the shape (Freyd \& Tversky, 1984). Second, symmetry about the top-bottom axis would provide redundant information identifying the top-bottom axis (i.e., in addition to the top and bottom features themselves). The effect of symmetry on orientation judgments was systematically examined here.
Two general predictions were made. First, reaction times (RTs) for the upright-nonupright conditions (Level 1) will be faster than those for the upside-downsideways conditions (Level 2). Second, Level 2 judgments will be faster for symmetrical than for asymmetrical figures, because symmetry about the top-bottom axis will enhance perception of that axis.

\section{Method}

Design. Two tasks were used, an upside-down task and a sideways task, each presented to a different group of subjects. Within each task, the effects of two nested conditions or levels were compared-an upright-nonupright condition and an upside-downsideways condition.

Subjects. Forty right-handed subjects from introductory psychology classes were assigned randomly to one of the two tasks. Fifteen females and 5 males received the upside-down task, and 19 females and 1 male received the sideways task. Data from 1 additional subject assigned to the upside-down task and 2 subjects assigned to the sideways task were discarded because of long reaction times (RTs), defined during performance on the first condition as more than three RTs over 1,000 msec and a mean RT over 2.5 standard deviations above the mean for that condition.

Apparatus and Stimuli. The stimuli were black line drawings of 20 familiar, mono-oriented shapes. Half of these were symmetrical front views: Christmas tree, face, flowers, house, jacket, lamp, pitcher, owl, snowman, and telephone. The other half were asymmetric side views. Of these, 5 faced left, car, cup, elephant, horse, sailboat, and 5 faced right, bicycle, cat, kangaroo, kettle, and truck. The long axes averaged $2.2 \mathrm{~cm}$, with a range of 1.6 to $2.6 \mathrm{~cm}$.

The stimuli were presented in a Gerbrands tachistoscope (Model T-3a). They appeared in the center of a square aperture, $8 \mathrm{~cm}$ on a side, at a viewing distance of $81 \mathrm{~cm}$, for an exposure duration of $300 \mathrm{msec}$. A fixation point appeared in the center of the field during the interstimulus interval. The stimulus field was adjusted to an intensity of $.39 \mathrm{fL}$, and the fixation field to $.05 \mathrm{fL}$ (as measured by a UDT Universal Optometer). Stimulus presentation was triggered by a signal from a TRS-80 Model III computer. Each stimulus presentation started an assembly language timing routine in the computer. A vocal response into a Sony microphone connected to the computer via a Lafayette voice relay (Model 1604A) terminated the timing routine.

Procedure. Two conditions were presented for each task. In one condition, an upright-nonupright identification was required, and in the other, identification of two nonupright orientations was required. For the subjects assigned to the "upside-down" task, the upright-nonupright condition consisted of upside-down and upright stimuli and the nonupright-nonupright condition consisted of upsidedown and sideways stimuli. For the subjects assigned to the "sideways" task, the comparable conditions consisted of sideways and upright stimuli, and sideways and upside-down stimuli. Upside-down stimuli were rotated $180^{\circ}$ in the picture plane, and sideways stimuli were rotated $90^{\circ}$ to the left. Throughout this paper, "turned to the left" refers to a $90^{\circ}$ tum counterclockwise and "turned to the right" refers to a $90^{\circ}$ turn clockwise.

The subjects were initially shown a sheet that displayed the set of 20 pictures in an upright orientation. Before each condition, the sheet was turned to each of the two positions in which the pictures would appear, and the subjects were allowed to look at the pictures for a few minutes. They were told to identify the orientation of the pictures to be presented with the word ("up," "'down," or "side") appropriate to the condition, and to respond as quickly and as accurately as possible.

At the beginning of a trial, the subject was asked to look at the fixation point in the center of an otherwise blank field. A single figure was presented, and the subject responded with the appropri- 
ate word. After stimulus termination, the fixation field reappeared. For each condition, 20 items were presented as practice (10 in each orientation) followed by 80 test trials, each item being presented twice in each orientation. Any errors were re-presented after 40 and 80 trials without the subject's knowledge. There was a short rest between conditions. The orientations for the second condition were then demonstrated with the presentation sheet. Twenty practice trials were again presented (10 in each orientation), followed by the same procedure used in the first condition.

The order of conditions was balanced across subjects. The stimuli were presented to each subject in a different random order, with the following constraints: (1) the same orientation did not appear on more than three consecutive trials, and (2) the two orientations appeared with equal frequency in the first and second halves of each condition.

After the main procedure had been completed, the subject rested for a few minutes, and then a second task was presented which permitted a comparison of the times required to activate the voice-relay for the three response words: "up," “down," and "side." Following the procedure used by Farrell (1979) and Sholl and Egeth (1981), the first letter of each of the spatial terms (U, D, and S) was presented in the tachistoscope for $300 \mathrm{msec}$, and the subject said the appropriate word ("up," "down," "side") as quickly as possible. After a practice set of 12 trials, 60 test trials were presented. Each spatial term appeared an equal number of times in a random sequence, with the constraint that the same letter would not appear more than three times in a row.

The RT to make each verbal response was measured because the responses of the subjects tested early in the procedure had suggested that "side" took longer to activate the relay than "up" or "down." For 20 of the 40 subjects, the second task and the main task were presented on different days because the second task was introduced only several days after testing had begun. The pattern of results was not affected by the intervention of this delay. The work of Farrell (1979) and of Sholl and Egeth (1981) had already shown that RTs were equal for articulation of the words "up," "down," "left," and "right."

\section{Results and Discussion}

First, RTs to the letters U, D, and S were analyzed in order to correct for any differences in the time it took for the response words to activate the relay. Since the RTs for the upside-down and the sideways subject groups were virtually identical, their data were combined. For the combined group, the relay was activated more slowly by "side" ( $508 \mathrm{msec}$ ) than by "up" ( $459 \mathrm{msec}$ ) or "down" (465 msec), which did not differ from each other. An analysis of variance (ANOVA) for the combined groups revealed a significant effect of word $[F(2,78)=76.49$, $p<.00001]$.
Because of this word effect, the RTs to make shapeorientation judgments were adjusted for each individual by subtracting the subject's time to say each word from the subject's total judgment time for each orientation. These adjusted scores were used in the statistical analyses.

However, the mean RTs presented in Table 1 are not the adjusted scores used in the analyses, but are scores that are comparable to those presented in subsequent experiments (in which the word "side" was not used). For the "up" and "down" RTs, the means of the raw scores are presented. For the "side" RTs, a correction was made which took into account the increased time required for the sibilant to activate the relay. That is, the mean difference in time to say "side" and "down," or "side" and "up" (depending on the condition) was subtracted from the raw mean RTs for " side" for that particular condition.

Upside-down task. The mean RTs for the upside-down task are shown in the upper part of Table 1. In the ANOVA, the within-subjects factors were orientation (upside down, other), level (down with up and down with side), and symmetry (symmetric, asymmetric), and order of levels was a between-subjects factor. Significant effects were exerted both by level $[F(1,18)=25.54$, $p<.001]$ and by symmetry $[F(1,18)=5.85, p<.02]$. However, there was a significant interaction between symmetry and level $[F(1,18)=32.61, p<.0001]$, since symmetrical items were relatively easier for the downside contrast but harder for the down-up contrast (see Table 1). Level also interacted with orientation $[F(1,18)=$ $11.95, p<.01$ ], because down judgments were slightly slower than up judgments in the down-up contrast, but slightly faster than side judgments in the down-side contrast. However, RTs in the down-side contrast were slower than those in the down-up contrast over and above the interactions with symmetry and orientation. Order interacted with orientation $[F(1,18)=5.52, p<.05]$, but the selective effect of practice was small and uninterpretable. The mean number of errors was less than 1 over the 160 test trials, and the errors paralleled RTs.

Sideways task. The mean RTs for the sideways task are presented in the lower part of Table 1 , and the data were analyzed in the same way as for the upside-down group. Again, level exerted a significant effect $[F(1,18)$ $=42.68, p<.0001]$; as anticipated, faster RTs appeared for the upright-nonupright contrast than for the contrast

Table 1

Mean Reaction Times (in Milliseconds) by Level, Orientation, and Symmetry for Experiment 1

\begin{tabular}{|c|c|c|c|c|c|c|c|}
\hline \multirow[b]{2}{*}{ Task } & \multirow[b]{2}{*}{ Stimuli } & \multicolumn{3}{|c|}{ Level 1} & \multicolumn{3}{|c|}{ Level 2} \\
\hline & & Down & Up & Combined & Down & Side* & Combined \\
\hline \multirow[t]{2}{*}{ Upside Down } & $\begin{array}{l}\text { Asymmetrical } \\
\text { Symmetrical } \\
\text { Combined }\end{array}$ & $\begin{array}{l}573 \\
592 \\
583\end{array}$ & $\begin{array}{l}557 \\
578 \\
568\end{array}$ & $\begin{array}{l}565 \\
585 \\
575\end{array}$ & $\begin{array}{l}639 \\
612 \\
626\end{array}$ & $\begin{array}{l}674 \\
619 \\
647\end{array}$ & $\begin{array}{l}657 \\
616 \\
637\end{array}$ \\
\hline & & Side* & Up & Combined & Side* & Down & Combined \\
\hline Sideways & $\begin{array}{l}\text { Asymmetrical } \\
\text { Symmetrical } \\
\text { Combined }\end{array}$ & $\begin{array}{l}600 \\
585 \\
593 \\
\end{array}$ & $\begin{array}{l}604 \\
570 \\
587\end{array}$ & $\begin{array}{l}602 \\
578 \\
590\end{array}$ & $\begin{array}{l}682 \\
638 \\
660 \\
\end{array}$ & $\begin{array}{l}656 \\
619 \\
638 \\
\end{array}$ & $\begin{array}{l}669 \\
628 \\
649 \\
\end{array}$ \\
\hline
\end{tabular}

*These RT values were corrected for the increased time to say "side" than "down" or "up" by subtracting the difference between these times (side-down or side-up) from the raw values for side. 
involving two nonuprights. Symmetrical figures facilitated orientation judgments for both levels $[F(1,18)=75.49$, $p<.0001]$ in this task. A marginally significant interaction appeared for level $\times$ orientation $\times$ order $[F(1,18)$ $=4.44, p<.05]$ that reflects small differences in opposite directions with no clear interpretation. Again, the mean number of errors was less than 1 over the 160 trials and paralleled RTs.

Symmetry. The effects of symmetry support the view that judgments of upside down and sideways were based on identifying the orientation of the top-bottom axis as either vertical or horizontal. For asymmetrical figures, this axis was defined only by the top and bottom features of the figure, whereas for symmetrical figures, the topbottom axis was defined both by features and by the line of symmetry. (For many of these front-view, symmetrical figures, although not all, the symmetrical axis was also the longer axis; we return to this point in the general discussion.) The redundant information defining the topbottom axis for symmetrical figures, as well as the rapid identification of symmetry (Freyd \& Tversky, 1984), would facilitate identification of the orientation of the axis, and hence of the orientation of the shape.

Somewhat unexpectedly, symmetry also facilitated judgments in the sideways-upright contrast. Although it could be argued that these judgments were also made by identifying the orientation of the top-bottom axis as vertical or horizontal, it is clear that other factors were at work because the judgment times for that contrast were so much faster than those for the sideways-upside-down contrasts. For the sideways-upright contrast, the perception of uprightness could have been facilitated by the vertical axis of symmetry, since symmetry about the vertical axis contributes to the perception of uprightness for geometric shapes (Ghent, 1961; Schaller \& Harris, 1975). This interpretation is consistent with the results for the upright-upside-down contrast, in which judgments were more difficult for the symmetrical, than for the asymmetrical, figures. In this case, symmetry about the vertical axis would contribute to the appearance of uprightness for the upside-down figures, increasing the difficulty in discriminating between these two orientations, and thus raising the RTs for both upright and upside down (see Table 1).

In summary, the results of this experiment support the predictions outlined earlier. First, the RTs for the uprightnonupright contrasts (Level 1) were faster than the RTs for the nonupright-nonupright contrasts (Level 2). Second, the orientations of symmetrical figures were identified more quickly than those of asymmetrical figures for the Level 2 conditions (upside-down-sideways). In addition, symmetry played a role in the upright-nonupright contrasts that was consistent with the proposed model.

\section{EXPERIMENT 2}

This experiment examined judgments at the second and third levels of difficulty in the model. Judgments that a shape is turned, for example, to the left, should be faster when the other orientation in the block of trials is upside down (Level 2) than when the other orientation involves shapes turned to the right (Level 3). However, both of these conditions are expected to yield RTs longer than those obtained for the upright-nonupright conditions of the previous experiment (Level 1). Again, symmetry would be expected to facilitate judgments in the left-down contrast (because these judgments are based on the orientation of the top-bottom axis), but not to facilitate judgments in the left-right contrast.

\section{Method}

Subjects. Twenty female and 4 male right-handed students between 17 and 21 years of age, drawn primarily from introductory psychology classes, took part in the experiment. Data from 2 additional subjects were discarded because of long RTs, defined during performance on the first condition as two or more RTs above 2,000 msec and mean RTs over 2.5 standard deviations above the mean for that condition.

Apparatus and Stimuli. The apparatus and stimuli were essentially the same as in Experiment 1, except for the orientation of the stimuli. The only other change from Experiment 1 was that (instead of computer control) each stimulus presentation triggered a Hunter Klockounter (Model $120 \mathrm{C}$ ). Calibration measurements showed that the Hunter Klockounter and the computer agreed to within $0.2 \%$. Again, the timing device was stopped by a vocal response into a Sony microphone connected to the Klockounter via a Lafayette voice relay (Model 1604A).

Procedure. Subjects were initially shown a sheet that displayed the full set of 20 pictures in an upright orientation. In one condition (Level 3), the subjects were told that each of the pictures would be presented in two orientations: rotated either $90^{\circ}$ to the left or $90^{\circ}$ to the right. They were to respond with the words "left"' (unlike Experiment 1, in which "side" was used) or "right," as appropriate, as quickly and accurately as possible. In the other condition (Level 2), the pictures were rotated either $90^{\circ}$ counterclockwise or $180^{\circ}$, the responses being "left" and "down," respectively. Before each condition, the sheet containing the pictures was turned to each of the two positions in which the figures would appear, and the subjects were allowed to look at the sheet for a few minutes. Again, there were 20 practice and 80 test trials for each condition.

As before, the order of conditions, or levels, was balanced across subjects, and the stimuli were presented in a different random order for each subject with the same constraints as used previously.

\section{Results and Discussion}

Reaction times were analyzed in a four-way ANOVA with orientation (left, other), symmetry (asymmetric, symmetric), and level (left with right and left with down) as within-subjects factors, and order (left-right contrast followed by left-down contrast, or vice versa) as a between-subjects factor. Significant main effects were ex-

Table 2

Mean Reaction Times (in Milliseconds) by Level, Orientation, and Symmetry for Experiment 2

\begin{tabular}{lccccccc}
\hline & \multicolumn{3}{c}{ Level 2 } & & \multicolumn{2}{c}{ Level 3 } \\
\cline { 2 - 4 } \cline { 7 - 8 } \multicolumn{1}{c}{ Stimuli } & Left & Down & Combined & & Left & Right & Combined \\
\hline Asymmetrical & 662 & 615 & 639 & & 698 & 694 & 696 \\
Symmetrical & 611 & 592 & 602 & & 682 & 686 & 684 \\
Combined & 637 & 604 & 621 & & 690 & 690 & 690 \\
\hline
\end{tabular}


erted by level $[F(1,22)=17.07, p<.001]$, orientation $[F(1,22)=4.60, p<.05]$, and symmetry $[F(1,22)=$ $5.20, p<.05]$ (see Table 2). In addition, level interacted with each of the other variables: with orientation $[F(1,22)$ $=11.85, p<.01]$, with order $[F(1,22)=5.26$, $p<.05]$, and marginally with symmetry $[F(1,22)=$ $4.14, p=.054]$. The error rate was low, averaging 1.5 over the 160 test trials, with an approximately equal number of errors for the two conditions.

As predicted, level exerted a marked effect on RT over and above its interaction with orientation: The RTs to left and down (Level 2) were both considerably faster than those to left and right (Level 3). Despite the fact that the subject made the same verbal response of "left" to the same pictures presented one at a time, the left judgments were made $53 \mathrm{msec}$ faster when the contrasting stimuli were upside down than when they were turned to the right [Bonferroni $t(1,23)=2.96, p<.05]$.

The interaction between level and orientation was due to the difference between orientations for the left-down contrast, whereas the two orientations yielded the same RTs in the left-right contrast. As can be seen in Table 2, the response to down was quicker than that to left [Bonferroni $t(1,23)=3.38, p<.02$ ]. A reliable difference between down and left (when right does not also appear in the condition) appears about half the time (Braine et al., 1981; Experiments 1, 2, and 4 of the present work). When symmetry and length of the vertical axis were systematically varied (in some unpublished work from our laboratory), it appeared that slower RTs to sideways than to upside down occurred only for sideview asymmetrical shapes with a short vertical axis (e.g., car), presumably because a $90^{\circ}$ tilt then produced shapes with a long vertical axis and a differentiated feature at one end (the front), which would be difficult to distinguish from upside-down shapes.

Order had a slight, nonsignificant effect in that the RTs were somewhat faster when a contrast level was presented second than when it was presented first. The order $\times$ level interaction resulted from the relatively greater improvement (when presented second) for the left-down contrast than for the left-right contrast.

As predicted, symmetry facilitated judgments in the leftdown contrast (see Table 2), replicating the results for the same contrasts in Experiment 1. Again, symmetry provided a redundant source of information for identifying the top-bottom axis, thus facilitating the down and left judgments. For the left-right condition, however, when attention presumably had to be directed to the ends of the horizontal axis, symmetry provided no additional information.

In summary, the present results provide additional support for the model described. Judgment of a shape as being turned to the left was made on different bases (as evidenced by the differences in RTs), depending on whether the contrasting orientation was upside down or turned to the right. Finally, a comparison of the absolute levels of RTs across the present and previous experiments shows a stepwise increase across the three degrees of complex- ity. The combined mean for the two upright-nonupright conditions of Experiment 1 was $582 \mathrm{msec}$; the combined mean for the three upside-down-sideways conditions of Experiments 1 and $2(637,649$, and $621 \mathrm{msec})$ was $635 \mathrm{msec}$, and the left-right mean from Experiment 2 was $690 \mathrm{msec}$.

\section{EXPERIMENT 3}

The next question is whether the three levels of difficulty also appear when subjects are presented with the four contrasting orientations: up, down, left, and right. The upright orientation should be identified most quickly because the judgment can be made that the shape looks rightly turned, in contrast to all the other stimuli, which look wrongly turned. Among the disoriented stimuli, down should be next in level of difficulty, since upsidedown shapes can be identified by the vertical orientation of the top-bottom axis of the shape, in contrast to the horizontal orientation of this axis for the sideways shapes. The orientations of shapes turned to the left and right should be most difficult, because the top feature must be related to a particular side of the spatial framework. Because the down judgments (in the context of sideways shapes) depend on identifying the orientation of the topbottom axis, these judgments should be faster for symmetrical than for asymmetrical shapes, as found in the previous two experiments.

\section{Method}

The apparatus, stimuli, and method were the same as for Experiment 1 , with the main exception being that four orientations were presented. The subjects were 17 female and 3 male right-handed college students between the ages of 17 and 22 years, again drawn from introductory psychology classes.

The subjects were to respond with the words "up," "down," "left," or "right," as appropriate, as quickly and accurately as possible. There were 40 practice trials, 10 in each orientation, followed by 160 test trials, with each item being presented twice in each orientation. Errors were re-presented after 40, 80, 120, and 160 trials without the subject's knowledge. There was a short rest after $\mathbf{8 0}$ trials. The stimuli were presented to each subject in a different random order with the same constraints as before.

\section{Results and Discussion}

A two-way, repeated measures analysis of variance was carried out on the factors of orientation (up, down, left, and right) and symmetry (asymmetry, symmetry). Both factors had highly significant effects [for orientation, $F(3,57)=24.05, p<.0001$; for symmetry, $F(1,19)=$ $10.76, p<.01]$. The interaction between orientation and symmetry just failed to reach significance $[F(3,57)=$ $2.62, p<.06]$. The effects of orientation and symmetry can be seen in Table 3 .

Comparison of the overall means for each orientation showed that the up-down difference failed to reach significance $[t(1,19)=1.69, p<.20]$, whereas up and down were each significantly faster than left and right (Bonferroni $t \mathrm{~s}$ were significant at less than the .01 level 
Table 3

Mean Reaction Times (in Milliseconds) by Orientation and Symmetry for Experiment 3

\begin{tabular}{lccccc}
\multicolumn{1}{c}{ Stimuli } & Up & Down & Left & Right & Combined \\
\hline Asymmetrical & 731 & 768 & 873 & 860 & 808 \\
Symmetrical & 720 & 725 & 827 & 820 & 773 \\
Combined & 726 & 747 & 850 & 840 & 791 \\
\hline
\end{tabular}

in all cases). The status of the up-down difference is unclear at this point, since the data suggest that there was an interaction with symmetry. That is, down judgments took $37 \mathrm{msec}$ longer than up judgments for asymmetrical shapes $[t(1,19)=2.75, p<.02]$, whereas the down judgments took only $5 \mathrm{msec}$ longer for the symmetrical shapes $(t=0.36)$. The role of symmetry as a factor influencing up-down differences in the context of more than two contrasting orientations will be examined and discussed further in the next experiment.

The error rate was again very low, averaging 1.2 over the 160 test trials. Almost all the errors were made on the left and right judgments, with only 2 errors being made to the up and down stimuli.

The results of the present experiment differ in two respects from those of Experiments 1 and 2. First, symmetry played a more widespread role than had been anticipated, affecting left and right as well as down judgments. Again, the more pervasive effect of symmetry can be explained by considering the role of the top-bottom axis in the particular contrasts provided in this experiment. For the left-right judgments of Experiment 2, the subject had only to attend to the ends of the horizontal axis since all stimuli were turned to the side. In the four-choice task, on the other hand, only half the shapes were turned to the side. Identifying these sideways shapes would be easier for symmetrical shapes than for asymmetrical ones because the top-bottom axis of the symmetrical shapes was defined both by features and by symmetry.

The second way in which the results of Experiment 3 differ from those of Experiments 1 and 2 is that the absolute levels of RT were about $150 \mathrm{msec}$ longer. The most plausible interpretation is that identifying one of two alternatives takes less time than identifying one of four alternatives. The effect of number of alternatives could operate at the perceptual end, the response end, or both.

In summary, the order of RTs for this set of orientation contrasts was as predicted: up was fastest, down was next (although there is some question concerning the status of the up-down difference), and left and right were considerably longer and of equal difficulty. Symmetry had a widespread effect on judgment times; its effects could be accounted for by assuming that symmetry facilitated the perception of the top-bottom axis of the shape, which then aided particular judgments.

\section{EXPERIMENT 4}

Three issues raised in the previous experiment were investigated here. First, the role of symmetry on up-down judgments in a multicontrast task was examined again. Will an up-down difference appear only for asymmetric figures, replicating the results of the previous experiment? Second, if the absolute levels of RT are related to the number of choices, will RTs for a three-choice task fall between those for the two- and four-choice tasks of previous experiments? Finally, it is possible that the RTs to upright figures were relatively long in Experiment 3 because the probability of occurrence of an upright figure was only one in four (whereas nonupright figures occurred three times as frequently). Would increasing the probability to one in three decrease the RT to upright orientations relative to other orientations? In order to present only three choices, and also to focus on the up and down judgments, figures were presented in up, down, and left orientations.

\section{Method}

The method followed the pattern of the previous experiments. Sixteen female and 4 male right-handed subjects were drawn from introductory psychology classes and ranged in age from 17 to 25 years. The apparatus and stimuli were as in Experiment 1, except that the pictures were presented in three orientations: upright, upside down, or rotated $90^{\circ}$ to the left, and the subjects responded accordingly with the words "up," "down," or "left"' as quickly and accurately as possible. There were 30 practice trials, 10 in each orientation, followed by 120 test trials, each item being presented twice in each orientation. There was a short rest after 60 trials, and errors were re-presented after 60 and 120 trials. Random ordering with constraints was carried out as before.

\section{Results and Discussion}

A two-way, repeated-measures ANOVA was done with orientation (up, down, and left) and symmetry (asymmetric, symmetric) as the main factors. Although both factors exerted significant effects, the significant interaction between the two $[F(2,38)=8.94, p<.001]$ best describes the data (Table 4). This interaction replicates the effect of symmetry on up-down judgments found in Experiment 3. That is, there were significant differences for the asymmetric figures between up and down [Bonferroni $t(1,19)=4.39, p<.01]$ and between up and left [Bonferroni $t(1,19)=3.96, p<.01$ ], but no significant differences for the symmetric figures. Down and left were not significantly different for either set of figures. The error rate was very low, averaging 1.1 out of 120 trials, and generally paralleled the RTs.

The effect of symmetry on judgment times can be accounted for by considering again the role played by the top-bottom axis and by assuming that symmetry is processed before orientation is judged. As discussed previously, the top-bottom axis was defined redundantly for

Table 4

Mean Reaction Times (in Milliseconds) by Orientation and Symmetry for Experiment 4

\begin{tabular}{lcccc}
\hline \multicolumn{1}{c}{ Stimuli } & Up & Down & Left & Combined \\
\hline Asymmetrical & 710 & 767 & 781 & 753 \\
Symmetrical & 727 & 728 & 728 & 728 \\
Combined & 719 & 748 & 755 & 741 \\
\hline
\end{tabular}


the symmetric figures by the features of the shape, by the line of symmetry, and, in many instances, by the long axis. The symmetric shapes could be divided into two orientation categories on the basis of the orientation of the line of symmetry as horizontal or vertical. For this set of three alternatives, a horizontal line of symmetry would uniquely signal a left-turned shape, providing a basis for the rapid identification of this orientation. Figures with a vertical line of symmetry could be identified as looking upright or nonupright (Level 1). This argument gives no advantage to the perception of uprightness for the symmetrical figures. A similar analysis could account for the equal RTs for upright and upside-down judgments of symmetrical figures in the four-choice task of the previous experiment. Symmetrical figures would be seen as having either a vertical or a horizontal line of symmetry, and for those with a vertical line of symmetry, orientation would be judged on the basis of uprightness and nonuprightness. That is, symmetry changed upside-down judgments in a multicontrast set from a Level 2 judgment to a Level 1 judgment.

For the asymmetrical figures, on the other hand, there would be no basis for an early segregation of shapes by orientation of the top-bottom axis and orientation judgments would be made in the way described by the model. Uprightness would be identified on a Level 1 basis; upside down and sideways would be Level 2 judgments based on identification of the orientation of the top-bottom axis of phenomenally nonupright shapes.

In addition to examining the relationship between orientation and symmetry, the present study was also concerned with the effects of number of choices on RTs. Decreasing the choices from four to three had no effect on RT for the judgments that were comparable. In the present experiment, the up and down RTs were 719 and $748 \mathrm{msec}$, respectively; the comparable RTs for the fourchoice experiment were 725 and $746 \mathrm{msec}$, combining over symmetry groups in both cases. The "left" responses in the two tasks cannot be compared, because a left judgment in the present three-choice task is assumed to be a sideways judgment.

\section{GENERAL DISCUSSION}

The present work has examined a particular conception of how the orientation of shape is judged-namely, that judgments may be made on various bases, which range from broad and imprecise, such as nonupright, to precise encodings of the relationship between a feature and the spatial framework. Support for this view has been obtained in the experiments reported here. The nature of the judgments made depended on the contrasts available, consistent with Garner's (1974) general claim that identifications of shapes or attributes are always made with respect to a set of possible stimuli.

Can the pattern of RTs found in the four experiments be accounted for other than in terms of the orientation judgments described here? One might argue that the RTs derive from the time required to recognize the stimulus rather than from the time required to judge its orientation. There are several lines of evidence against this interpretation. First, the results reported show that there is no simple ordering of the difficulty of identifying orientations. For example, upside down was as easy as upright when the two were contrasted, but increased significantly in difficulty when sideways figures were introduced, yet the recognizability of the upside-down shapes, presented one at a time, should not have been affected by the orientation of other stimuli. Second, the literature on recognizability of shapes has not found consistent differences among orientations except for reliably better recognition for upright figures (e.g., Braine, 1965; Jolicoeur \& Landau, 1984; Rock, 1973). In both the present and previous work (Braine et al., 1981), upright judgments were not significantly faster than upside-down ones for Level 1 comparisons. Finally, Maki (1986) recently investigated recognition of shape with many of the shapes used here, and found that $90^{\circ}$ rotations tended to yield somewhat faster recognition times than did $180^{\circ}$ rotations, which was not the pattern of our results.

Symmetry. Symmetry often facilitated judgment times. However, this effect was not a general one in the present experiments, and in previous work with a verification method, symmetry had no effect at all on orientation judgments (Maki \& Braine, 1985). Thus, the facilitation of orientation judgments by symmetry cannot be due simply to a faster encoding of symmetric stimuli. In the present work, judgments were facilitated significantly only when perception of the orientation of the top-bottom axis contributed to the identification of orientation, and in these instances, symmetry provided redundant information defining the top-bottom axis. That is, symmetry did not facilitate particular kinds of orientation judgments, but its effect depended on the set of comparison orientations.

The subtlety of the symmetry effects becomes evident when the times to make "up" and "down" judgments in Experiment 1 (see Table 1) are compared with those of Experiments 3 and 4 (see Tables 3 and 4). These judgments were slowed by symmetry in Experiment 1, but facilitated by symmetry in the other experiments. Since the upright and upside-down figures presented in Experiment 1 (Level 1 judgment) both had a vertical line of symmetry, and since a vertical line of symmetry contributes to the perception of uprightness (Ghent, 1961; Schaller \& Harris, 1975), symmetry would contribute to the appearance of uprightness for the upside-down figures, thus increasing the difficulty of identifying each orientation. In Experiments 3 and 4, on the other hand, symmetry facilitated the upside-down judgment by providing a basis for dividing the orientations into two groups, those with a vertical and those with a horizontal line of symmetry. The figures with a vertical line of symmetry could be judged as upright or nonupright, permitting a Level 1, instead of a Level 2 , judgment to be made.

The effects of symmetry point to an aspect of the model that was not previously apparent. When upright, upside- 
down, and sideways (left and/or right) stimuli are presented in the same task, RTs to upside-down stimuli were predicted to take longer than those to upright stimuli, on the grounds that identifying an item as being specifically upside down requires identifying the figure as nonupright, with the top-bottom axis in a vertical orientation. However, it is only in the case of asymmetrical figures that this requirement need be met. For symmetrical figures, as previous discussions have noted, the axis of symmetry provides a basis for changing an upside-down judgment in a multicontrast task from a Level 2 to a Level 1 judgment. The model described in the introduction applies directly to asymmetrical stimuli, whereas some adjustment in predictions must be made for figures that are symmetrical about the top-bottom axis with some combinations of contrasts.

Nature of judgments. The orientation judgments examined are categorical judgments by definition (upright, sideways, upside down, left, right), and it is clear from the data that, as anticipated, angular differences between stimuli $\left(90^{\circ}\right.$ or $\left.180^{\circ}\right)$ did not affect RT. In most instances, the angular differences between stimuli bore no relationship to RT (Experiments 1, 3, and 4), and in Experiment 2, the shorter RTs were associated with the condition involving the smaller angular difference between the stimuli. Moreover, the pattern of RTs found here is not a function of presenting only orientations that fall along the vertical and horizontal axes, since a similar pattern appears for identifying the orientations of alphanumeric shapes rotated $60^{\circ}, 120^{\circ}$, and $180^{\circ}$ from the upright (Corballis, Zbrodoff, Shetzer, \& Butler, 1978). Although these authors explicitly assume that orientation is a dimension that is phenomenally continuous (p. 104), their data lend themselves better to the view that the judgments were categorical. Judgment times for the positive detection of the orientation of normal letters were fastest for upright, next for upside down, and slowest (in most cases) for the $60^{\circ}$ and $120^{\circ}$ rotations. This pattern fits well with the model proposed, and does not show a consistent relationship to degree of tilt.

Some readers have suggested that the present results have to do with discrimination among orientations, but not with the identification of orientation. There are several reasons for rejecting this interpretation. First, the procedure used was not a discrimination method, which ordinarily requires a choice to be made between two stimuli presented simultaneously or successively. In the present work, the orientation of each stimulus, presented one at a time, was identified, or categorized, as in other work on the identification of shape orientation (e.g., Farrell, 1979; Maki et al., 1979). Furthermore, subjects had to base their judgments on very general attributes of the shapes (e.g., nonuprightness, leftness), and it is hard to see how the abstraction of such properties could be construed as being different from identification (at different degrees of precision) of the orientation of the shape. Finally, and most importantly, it is not possible, to our minds, to make "pure" judgments of orientation. We be- lieve, as Garner (1974) has suggested for other problems, that the orientation of a singly presented shape is always judged with respect to an implicit (if not an explicit) set of other orientations. In any case, it is unclear how judgments of orientation could be investigated without the presentation of contrasting orientations (unless only one judgment of one stimulus were obtained from one subject at a time, a task that would nevertheless raise many problems of interpretation).

Left-right identifications. There are two points to be made with respect to the relative difficulty of left-right orientation judgments. First, the present work offers an interpretation that does not focus on the symmetry of the organism (e.g., Corballis \& Beale, 1976) or the characteristics of the horizontal axis (e.g., Farrell, 1979). Leftright judgments require an encoding of the relationship between a feature of a phenomenally sideways shape and a framework, and this relational judgment is assumed to be cognitively more complex than the match-mismatch process assumed to underlie upright-nonupright judgments. ${ }^{2} \mathrm{~A}$ focus on the cognitive aspects of the judgment, rather than on characteristics of the horizontal and vertical axes, is compatible with work referred to in the introduction indicating that the difficulty of left-right orientation judgments is very sensitive to task factors, and that it is questionable to what extent the horizontal axis is even a critical factor in left-right difficulties (see also Rock et al., 1981).

Second, the claim has been made that a greater difficulty in making left-right than up-down judgments is tied to the use of spatial terms and not to the nature of the spatial judgments required (Maki et al., 1979; Sholl \& Egeth, 1981). The results of the experiments reported here indicate that the time to respond was not related to the use of a particular spatial label, but was related to the particular judgment required by the task. For example, the time to give the verbal judgment of "left" in the context of upside-down orientations was different from that needed in the context of another sideways orientation, presumably because of the different kinds of judgments required under these conditions. It is clear that a particular spatial label is not associated with a specific spatial concept or underlying judgment, at least for judgments of shape orientation. These data, added to other results (Maki \& Braine, 1985), show that left-right difficulties in adults are not simply a function of the use of spatial terms.

Conclusions. This work has both procedural and theoretical implications for the investigation of shape orientation judgments. From a procedural point of view, any effort to elicit specifically upside-down judgments (in contrast to nonupright judgments) requires the presentation of upside-down and sideways stimuli, rather than the usual pairing of upside-down and upright stimuli (e.g., Farrell, 1979; Maki et al., 1979; Sholl \& Egeth, 1981). Furthermore, symmetry (with respect to the top-bottom axis) needs to be explicitly controlled and examined, because the nature of a subject's task may be somewhat different with symmetrical and asymmetrical shapes; for example, 
a four-choice task (as in Experiment 3) may be treated as two two-choice tasks for symmetrical shapes, but not for asymmetrical shapes.

From a theoretical point of view, the proposed model fills a gap in the literature by providing an explicit description of how different judgments of shape orientation may be made. Two unique features of the model are (1) that judgments are made in different ways that vary in the specificity (and, consequently, the difficulty) of the judgment, and (2) that a normative position is part of the system of orientation judgments for shapes. It is claimed that shape orientation judgments tend to be categorical (unless precluded by the task), that different bases for judgment may underlie the use of the same spatial words, and that sets of contrasting orientations can be arranged in an absolute order of difficulty, as described by the model's three levels, but that individual orientation judgments themselves cannot be so arranged. Finally, the greater difficulty found for left-right than for upright-nonupright judgments has been interpreted as being due to the different cognitive bases used in making these judgments.

\section{REFERENCES}

Antonovsky, H. F., \& Ghent, L. (1964). Cross-cultural consistency of children's preferences for the orientation of figures. American Journal of Psychology, 77, 295-297.

ARNHEIM, R. (1954). Art and visual perception. Los Angeles: University of California Press.

AtTNEAve, F., \& OLson, R. K. (1967). Discriminability of stimuli varying in physical and retinal orientation. Journal of Experimental Psychology, 74, 149-157.

Braine, L. G. (1965). Disorientation of forms: An examination of Rock's theory. Psychonomic Science, 3, 541-542.

BraINE, L. G. (1978). A new slant on orientation perception. American Psychologist, 33, 10-22.

Braine, L. G., \& KNox, C. (1975). Children's orientation judgments: Retinally or environmentally determined? Perception \& Psychophysics, $17,473-479$.

Braine, L. G., Lerner, C., Relyea, L. (1980). Levels in the identifying of orientation by preschool children. Journal of Experimental Child Psychology, 30, 171-185.

Braine, L. G., Relyea, L., \& Davidman, L. (1981). On how adults identify the orientation of a shape. Perception \& Psychophysics, 29, 138-144.

COOPER, L. A., \& ShEPARD, R. N. (1973). Chronometric studies of the rotation of mental images. In W. G. Chase (Ed.), Visual information processing. New York: Academic Press.

Corballis, M. C., Beale, 1. L. (1976). The psychology of left and right. Hillsdale, NJ: Erlbaum.

Corbalus, M. C., Zbrodoff, N. J., Shetzer, L. I., Butler, P. B. (1978). Decisions about identity and orientation of rotated letters and digits. Memory \& Cognition, 6, 98-107.

FaGAN, J. F., \& Shepherd, P. A. (1979). Infants' perception of face orientation. Infant Behavior \& Development, 2, 227-234.

FARRELL, W. S. (1979). Coding left and right. Journal of Experimental Psychology: Human Perception \& Performance, 5, 42-51.

Fisher, C. B. (1979). Children's memory for orientation in the absence of external cues. Child Development, 50, 1088-1092.

Fisher, C. B. (1982). The role of stimulus alignment in children's memory for line orientation. Child Development, 53, 1070-1074.

FREYD, J., \& TVERSKY, B. (1984). Force of symmetry in form perception. American Journal of Psychology, 97, 109-126.
GARNER, W. R. (1974). Processing of information and structure. Hillsdale, NJ: Erlbaum.

GHENT, L. (1961). Form and its orientation: A child's-eye view. American Journal of Psychology, 74, 177-190.

Greene, S. L., Plastow, E., \& Braine, L. G. (1985). Judging the location of features of naturalistic and geometric shapes. Perception \& Psychophysics, 37, 148-154.

HowARD, I. P. (1982). Human visual orientation. New York: Wiley. HUTTENLOCHER, J. (1967). Discrimination of figure orientation: Effects of relative position. Journal of Comparative \& Physiological Psychology, 63, 359-361.

JolicoeUR, P., LANDAU, M. J. (1984). Effects of orientation on the identification of simple visual patterns. Canadian Journal of Psychology, 38, 80-93.

MAKI, R. H. (1986). Naming and locating the tops of rotated pictures. Canadian Journal of Psychology, 40, 368-387.

MAKI, R. H., BRAINE, L. G. (1985). The role of verbal labels in the judgment of orientation and location. Perception, 14, 67-80.

Maki, R. H., Grandy, C. A., \& HaUge, G. (1979). Why is telling right from left more difficult than telling above from below? Journal of Experimental Psychology: Human Perception \& Performance, 5 , $52-67$

Pick, H. L., Klein, R. E., \& PICK, A. D. (1966). Visual and tactual identification of form orientation. Journal of Experimental Child Psychology, 4, 391-397.

Rock, I. (1973). Orientation and form. New York: Academic Press. Rock, I., Di ViTA, J., \& BARBEITo, R. (1981). The effect on form perception of change of orientation in the third dimension. Journal of Experimental Psychology: Human Perception \& Performance, 7, 719-732.

SCHALlER, M. J., \& HARRIS, L. J. (1975). "Upright" orientations of forms change with subject age and with features of form. Perception \& Psychophysics, 17, 179-188.

SHOLL, M. J., EGETH, H. E. (1981). Right-left confusion in the adult: A verbal labeling effect. Memory \& Cognition, 9, 339-350.

STAller, J., \& SEKUler, R. (1976). Mirror-image confusion in adults and children. American Joumal of Psychology, 89, 253-268.

VoGEL, J. M., BEHREND, D. (1982, November). Adults' processing of orientation: Left-right is harder than up-down. Paper presented at the meetings of the Psychonomic Society, Minneapolis.

\section{NOTES}

1. There is a subtle point to be made here concerning the role of a spatial referent system in the different orientation judgments. Spatial judgments always have to be made with respect to a framework. A postulated match-mismatch process must assume that the same spatial framework is being used when the two items are compared; the perception of verticality or horizontality is not absolute, but occurs within an implied framework. Nevertheless, these Level 1 and Level 2 judgments are judgments about a perceived property of the object, and not about the relationship between the shape and the framework. On the other hand, the judgment that a feature of a shape is in a specific location is a judgment that describes a relationship between the framework and the shape. The latter judgment is seen as a framework judgment, because the shape is related to the framework, although a spatial framework necessarily functions in the other judgments as well.

2. The possible role of the framework in the difficulty of making leftright judgments was explored in another series of experiments (Greene, Plastow, \& Braine, 1985). Although the sides of the framework influenced the difficulty of identifying the location of a dotted line in doubly symmetrical shapes, this effect of the spatial framework was easily overshadowed by the orientation properties of natural shapes.

(Manuscript received July 21, 1986; revision accepted for publication November $28,1986$. 